\title{
VIDROS RECOBERTOS COM CAMADAS DELGADAS TRANSPARENTES DE SnO
}

\author{
Alessandro P. Rizzato, Sandra H. Pulcinelli e Celso V. Santilli \\ Instituto de Química - UNESP - CP 355 - 14 801-970 - Araraquara - SP
}

Recebido em 2/12/96; aceito em 2/6/97

\begin{abstract}
GLASSES COATED WITH TRANSPARENT THIN LAYERS OF $\mathrm{SnO}_{2}$. $\mathrm{SnO}_{2}$ thin layers, prepared from aqueous colloidal suspensions by the sol-gel process, have been dip-coated on commercial borosilicate glasses. The effect of the conditions of deposition on the optical and structural characteristics of the thin layers was analysed by UV-Vis spectroscopy, x-ray reflectometry and electron scanning microscopy. Layers prepared with withdrawal speed in between 0.1 and $10 \mathrm{~cm} / \mathrm{min}$ show thickness smaller than $90 \mathrm{~nm}$, roughness of the order of $2 \mathrm{~nm}$ and transmittance higher than $80 \%$, resulting in good optical quality samples. The roughness increases from 2 to $11 \mathrm{~nm}$ as the withdrawal speed increases from 10 to $80 \mathrm{~cm} / \mathrm{min}$, what seems to be associated to the enlargement of the layers thickness $(>90 \mathrm{~nm})$. The measurements of mass loss, done after etching with fluoridric acid show that the coated samples are more corrosion resistant than the uncoated borosilicate glass.
\end{abstract}

Keywords: $\mathrm{SnO}_{2}$ thin layers; corrosion resistant; coated borosilicate glass.

\section{INTRODUÇÃO}

Vidros tradicionais recobertos com camadas delgadas (< 1000nm) transparentes de $\mathrm{SnO}_{2}$ apresentam maior resistência mecânica à fratura e ao desgaste abrasivo e químico. Este comportamento é explorado comercialmente na fabricação de garrafas e embalagens descartáveis mais baratas e mais leves (paredes com espessura reduzida), e com resistência à fratura equivalente às tradicionais ${ }^{1}$. Outra aplicação destas camadas delgadas é em utensílios de vidro empregados em restaurantes e no transporte e condicionamento de alimentos, que geralmente são submetidos a condições extremas de desgaste abrasivo e químico. Além disto, peças de vidro de elevado valor decorativo, por apresentar brilho iridiscente, são fabricadas com depósitos superficiais de $\mathrm{SnO}_{2}$ com espessura da mesma ordem de grandeza do comprimento de onda da luz visível (400 a 1000nm) ${ }^{2}$.

Por outro lado, o dióxido de estanho é praticamente insolúvel em $\mathrm{pH}$ entre 1 e 14 , o que permite prever um aumento na resistência ao ataque hidrolítico dos vidros a base de borossilicato e soda cáustica recobertos com $\mathrm{SnO}_{2}$. Este tipo de ataque é frequente nos vidros destinados a embalagem de soluções ou suspensões farmacêuticas, limitando o período de validade destes produtos. Tradicionalmente, a resistência hidrolítica dos vidros de soda cáustica é promovida pelo tratamento de desalcalinização superficial efetuado em presença de $\mathrm{SO}_{2}$ a aproximadamente $600^{\circ} \mathrm{C}^{3}$.

As camadas delgadas de $\mathrm{SnO}_{2}$ são, usualmente, depositadas pela nebulização de solução aquosa de $\mathrm{SnCl}_{4}$ sobre a superfície do vidro aquecido entre 500 e $600^{\circ} \mathrm{C}^{1,2}$. Neste processo ocorre formação de vapores extremamente tóxicos e corrosivos $\left(\mathrm{Cl}_{2}, \mathrm{HCl}\right)$, exigindo instalações especiais para tratamento de rejeitos gasosos para evitar danos ao meio ambiente e à saúde. Este tipo de problema tem sido contornado, em parte, pela utilização de compostos organoclorados a base de estanho, que por sua vez são classificados como cancerígenos ${ }^{2}$.

Uma rota alternativa para a deposição de camadas delgadas é o emprego do processo de dip-coating, onde o vidro é emergido de uma suspensão coloidal a uma velocidade controlada. Durante o movimento de emersão a suspensão é arrastada com o substrato, causando um aumento na área de evaporação e na

E-mail: sandrap@iq.uesp.br taxa de secagem, o que leva à formação de uma camada delgada constituída pelas partículas coloidais. Este método permite obter depósitos sobre substratos de grandes dimensões e de geometria complexa, com bom controle da espessura. Além disto, os equipamentos empregados são de simples manuseio e operam em condições próximas à ambiente ${ }^{4}$.

O objetivo deste trabalho é obter informações sobre os parâmetros experimentais que permitem controlar as características estruturais das camadas delgadas de $\mathrm{SnO}_{2}$ depositadas por dipcoating sobre vidros de borossilicato. A parte final do trabalho demonstra a eficiência das camadas delgadas de $\mathrm{SnO}_{2}$ na proteção contra o desgaste por dissolução hidrolítica dos vidros.

\section{EXPERIMENTAL}

As camadas delgadas foram depositadas sobre lâminas de vidro comercial (Corning) de borossilicato $(2 \mathrm{~cm}$ de largura por $4 \mathrm{~cm}$ de comprimento) por dip-coating empregando-se suspensões coloidais aquosas de $\mathrm{SnO}_{2}$. As condições de preparação destas suspensões a partir do processo sol-gel, bem como suas características físico-químicas e estruturais foram descritas em publicações anteriores $^{5-7}$. Visando estabelecer as condições de formação de depósitos homogêneos e com espessura controlada, as camadas delgadas foram preparadas com diferentes velocidades $(0,1 \leq \mathrm{v} \leq 90 \mathrm{~cm} / \mathrm{min})$ e números $(1 \leq \mathrm{n} \leq 30)$ de emersões, usando-se suspensões coloidais com concentração de $\mathrm{SnO}_{2}$ e de íons cloreto de $1,3 \mathrm{~mol} / \mathrm{L}$ e $10^{-5} \mathrm{~mol} / \mathrm{L}$, respectivamente. As amostras foram tratadas a $400^{\circ} \mathrm{C}$ por $2 \mathrm{~h}$.

A espessura, rugosidade e densidade aparente dos depósitos foram determinadas a partir das curvas de reflexão especular de raios x. Empregou-se uma câmara de reflexão especular acoplada ao difratômetro de pó convencional (Siemens, D5000). Para a aquisição dos dados foi utilizada fenda primária e no feixe refletido, de 2 e $0,6 \mathrm{~mm}$, respectivamente, e abertura do anteparo sobre a superfície da amostra de $0,01 \mathrm{~mm}$. O feixe refletido foi monocromatizado por filtro de $\mathrm{Ni}\left(\mathrm{CuK}_{\alpha}\right.$, $\lambda=1,5418 \AA$ ). Para cada amostra o alinhamento da superfície do depósito foi efetuado por rotação do porta-amostras em $\theta-2 \theta$, entre 0,1 e $6^{\circ}(2 \theta)$ e ajuste da geometria de modo a obter o máximo de reflexão. Os parâmetros estruturais das amostras foram calculados usando-se o programa REFSIM, que ajusta a equação de refletividade ${ }^{8}$ às curvas experimentais, pelo método de mínimos quadrados. 
A qualidade óptica das amostras foi avaliada a partir de medidas de transmitância na região do UV-Vis, usando-se um espectrofotômetro HP8451 Diode Harray.

$\mathrm{O}$ efeito do recobrimento com $\mathrm{SnO}_{2}$ no aumento da resistência ao ataque químico foi avaliado a partir de medidas de perda de massa em função do tempo de imersão das amostras em $200 \mathrm{~mL}$ de uma solução aquosa de ácido fluorídrico (2mol/ $\mathrm{L}, \mathrm{pH}<1)$. A superfície das amostras submetidas ao ataque químico foi observada por microscopia eletrônica de varredura empregando-se um equipamento Jeol JSM - T 330A.

\section{RESULTADOS E DISCUSSÃO}

\section{i) Características estruturais}

As curvas de transmitância na região do visível e ultra-violeta próximo das amostras preparadas com 10 e 30 emersões, obtidas a partir da diferença entre os espectros do substrato com e sem depósito, são apresentadas na figura 1. A transparência superior a $80 \%$ observada na região do visível ilustra a boa qualidade óptica das amostras. Na realidade, é impossível distinguir a olho nu uma lâmina de vidro com e sem recobrimento, o que evidencia a homogeneidade e a uniformidade da camada de $\mathrm{SnO}_{2}$ sobre toda a extensão do substrato. A forte absorção observada na região do ultra-violeta próximo $(\lambda<340 \mathrm{~nm})$ é típica da transição interbanda (valência-condução) em semi-condutores de banda larga ${ }^{9}$. No caso dos vidros de borossilicato a borda de absorção localiza-se em torno de $300 \mathrm{~nm}$, de modo que o recobrimento com as camadas delgadas de $\mathrm{SnO}_{2}$ praticamente não altera a transmitância do substrato na região do visível.

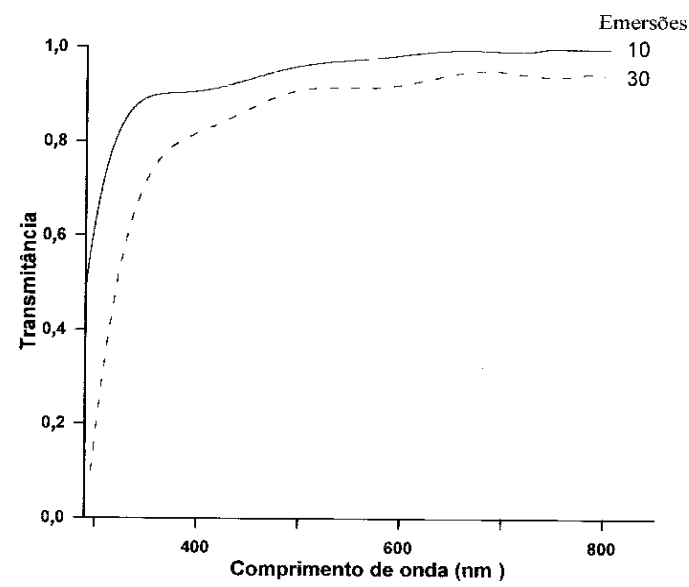

Figura 1. Transmitância na região do UV-Vis das camadas delgadas de $\mathrm{SnO}_{2}$ preparadas com dez e trinta emersões.

O efeito do número e da velocidade de emersão nas características estruturais das camadas delgadas pode ser avaliado a partir das curvas de reflexão especular de raios $\mathrm{x}$ apresentadas nas figuras 2 e 3 , respectivamente. A reflectividade é máxima (total) a baixo ângulo, e diminue de cinco ordens de grandeza acima de um valor angular crítico $\left(\theta_{c}\right)$. Este valor permite calcular a densidade aparente $(\rho)$ da camada delgada empregando-se a relação ${ }^{8}$ :

$$
\theta_{c}=\sqrt{C_{p}}
$$

onde C é uma constante que depende da natureza dos compostos e de parâmetros instrumentais. Os valores de densidade aparente calculados para as amostras preparadas em diferentes condições situam-se em torno de $3,6 \pm 0,1 \mathrm{~g} / \mathrm{cm}^{3}$. Este valor é muito inferior à densidade teórica do $\mathrm{SnO}_{2}\left(7,0 \mathrm{~g} / \mathrm{cm}^{3}\right)$, indicando que as camadas delgadas devem conter um grande volume de poros.

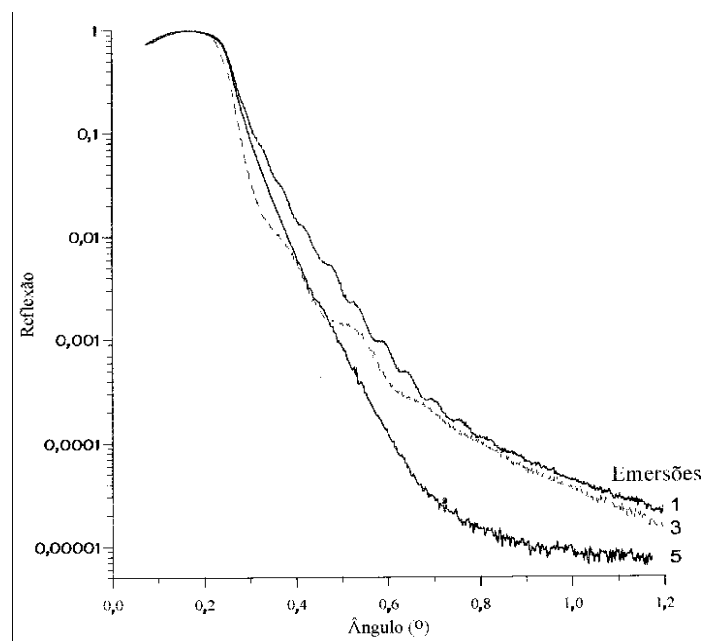

Figura 2. Curvas de reflexão especular de raio $x$ das camadas delgadas de $\mathrm{SnO}_{2}$ preparadas com uma, três e cinco emersões.

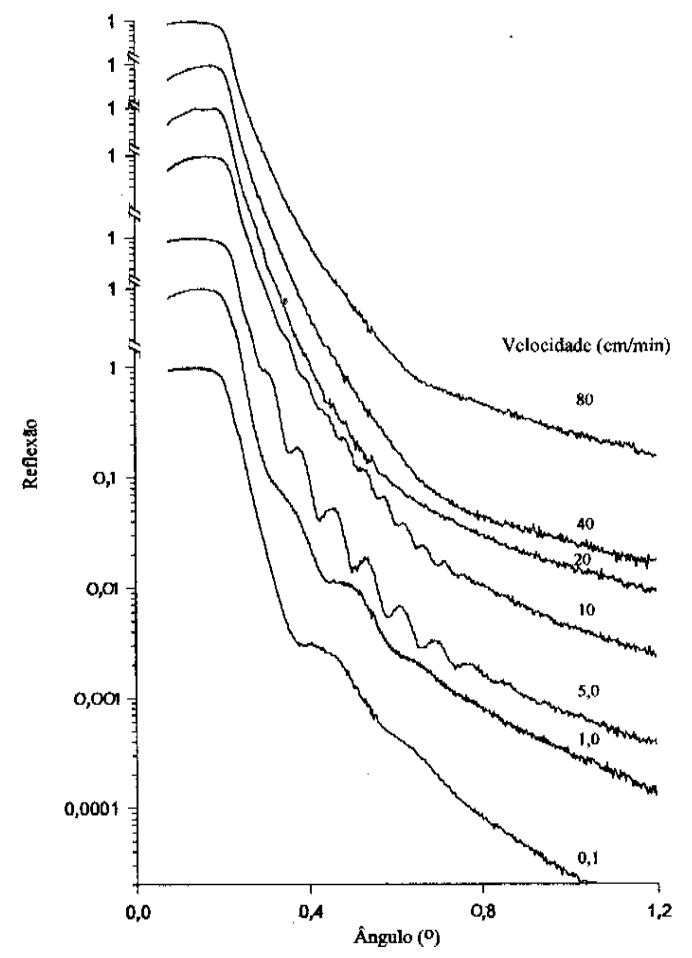

Figura 3. Evolução das curvas de reflexão especular de raios $x$ com a velocidade de emersão usada na preparação das camadas delgadas de $\mathrm{SnO}_{2}$.

Acima do ângulo crítico observa-se claramente a presença de franjas de interferência, características da espessura regular da camada delgada. A diminuição gradual da amplitude das franjas com o aumento do ângulo de reflexão é indicativo da rugosidade da superfície da camada delgada. Além disto, é interessante notar que as amostras preparadas com cinco ou mais emersões e com velocidade elevada $(\mathrm{v}>10 \mathrm{~cm} / \mathrm{min})$ não apresentam franjas de interferência. Este comportamento indica que nestas condições de preparação as camadas delgadas não são homogêneas, podendo apresentar falhas de deposição, regiões de desprendimento do substrato e variações consideráveis de espessura.

A tabela 1 agrupa os valores calculados para a espessura e rugosidade da superfície do depósito e da interface entre a camada delgada e o vidro de borossilicato das amostras prepara- 
das em diferentes condições. Conforme previsto pelos modelos clássicos de deposição por emersão ${ }^{4}$, o aumento do arraste viscoso com a velocidade de emersão favorece o aumento da espessura do filme. Deste modo, é possível controlar facilmente a espessura da camada depositada ajustando-se o número ou a velocidade de emersão. É interessante observar que a rugosidade da camada depositada praticamente não varia com as condições de deposição. Entretanto, a rugosidade da interface substrato-depósito aumenta continuamente quando a velocidade de emersão cresce de 10 para $80 \mathrm{~cm} / \mathrm{min}$; o mesmo comportamento é observado com o aumento do número de deposições. Isto sugere que a compatibilidade entre o depósito e o substrato diminue com a espessura da camada del-

Tabela 1. Influência das condições de preparação na espessura e rugosidade das camadas delgadas.

\begin{tabular}{cccc}
\hline & \multicolumn{2}{c}{ Rugosidade (nm) } \\
\hline $\begin{array}{c}\text { Número } \\
\text { de emersão }\end{array}$ & $\begin{array}{c}\text { Espessura } \\
(\mathrm{nm})\end{array}$ & $\begin{array}{c}\text { Superfície } \\
\text { das camadas } \\
\text { delgadas }\end{array}$ & $\begin{array}{c}\text { Interface } \\
\text { substrato- } \\
\text { depósito }\end{array}$ \\
\hline 1 & 20 & 0,8 & 2,0 \\
3 & 53 & 1,9 & 2,0 \\
5 & - & 2,2 & 9,5 \\
\hline Velocidade & & & \\
(cm/min) & & 1,0 & 1,6 \\
\hline 0,1 & 21 & 1,0 & 1,6 \\
1,0 & 29 & 1,9 & 1,5 \\
5,0 & 53 & 1,7 & 1,5 \\
10,0 & 90 & 1,9 & 3,4 \\
20,0 & - & 1,9 & 8,3 \\
40,0 & - & 1,9 & 11,5 \\
80,0 & - & & \\
\hline
\end{tabular}

gada.

As figuras $4 \mathrm{a}$ e $4 \mathrm{~b}$ mostram as micrografias da superfície das camadas delgadas preparadas com 3 e 10 emersões, respectivamente. Não foi possível observar contrastes associados aos aspectos topográficos na maioria da superfície das amostras preparadas com número e velocidades de emersões inferiores a 5 e $10 \mathrm{~cm} / \mathrm{min}$, respectivamente. Falhas de deposição semelhantes à observada na figura 4a são raras nas amostras preparadas nestas condições. Ao contrário, as nervuras e protuberâncias verificadas na fotomicrografia da figura $4 \mathrm{~b}$ estão presentes em toda a superfície das amostras preparadas com número e velocidades de emersão superiores a 5 e $10 \mathrm{~cm} / \mathrm{min}$, respectivamente. Esse tipo de defeito só é observado após o tratamento térmico a $400^{\circ} \mathrm{C}$, e sua formação pode estar associada às tensões resultantes da diferença de coeficiente de expansão térmica entre o substrato e a camada delgada. Obviamente, este fenômeno torna-se mais importante a medida que a espessura da camada delgada aumenta, podendo inclusive causar o desprendimento do depósito.

\section{ii) Resistência ao ataque por ácido}

O desempenho das camadas delgadas de $\mathrm{SnO}_{2}$ como barreiras protetoras contra o ataque químico dos vidros de borossilicato pode ser avaliado a partir dos resultados de perda de massa em função do tempo de exposição à solução de ácido fluorídrico, mostrados na figura 5. Os resultados ilustram o efeito do aumento do número de emersões, de 0 para 5, na dissolução do vidro. A comparação entre as curvas do vidro com e sem depósito superficial (número de emersão $=$ zero) mostra claramente uma diminuição significativa na taxa de dissolução das amostras recobertas. Além disto, a perda de massa diminue com a espessura da camada delgada, atingindo um mínimo para as amostras preparadas

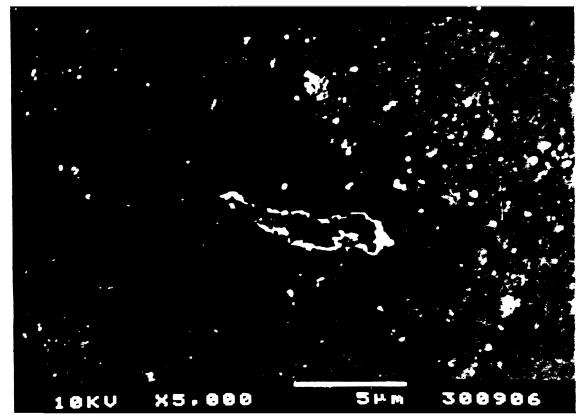

(a)

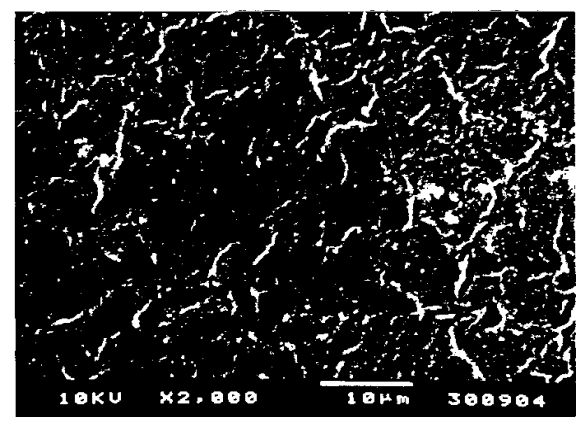

(b)

Figura 4. Micrografias obtidas por microscopia eletrônica de varredura da superfície da camada delgada preparada com 3 (a) e 10 (b) emersões.

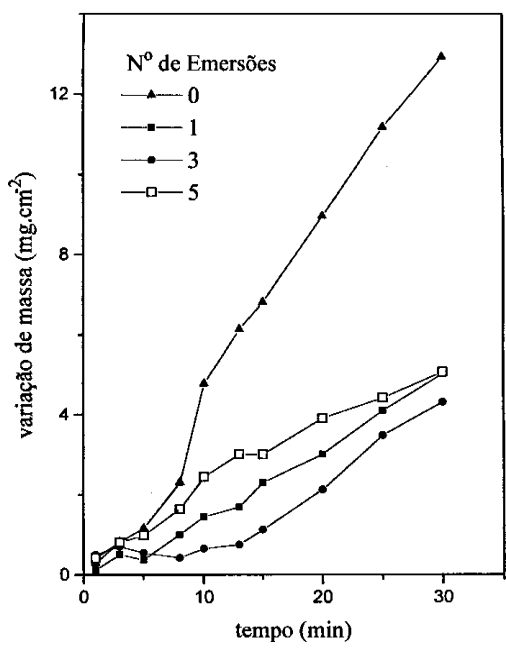

Figura 5. Curvas de variação de massa normalizada pela área da amostra em função do tempo de ataque pela solução aquosa de ácido fluorídrico.

com 3 emersões.

As fotomicrografias apresentadas nas figuras $6 \mathrm{a}$ e $6 \mathrm{~b}$ revelam, respectivamente, o aspecto da superfície do vidro de borossilicato comercial e da amostra recoberta com $\mathrm{SnO}_{2}$, ambas submetidas a $25 \mathrm{~min}$ de ataque por ácido fluorídrico. $\mathrm{O}$ ataque do vidro comercial causa a formação de partículas e poros em toda a extensão da superfície. Apesar do mecanismo de ataque por ácido fluorídrico não ser conhecido com precisão, a presença destas partículas é, geralmente, atribuída à dissolução do hidróxido de silício e reprecipitação de fluoretos ${ }^{3}$. Estas partículas são observadas em menor quantidade nas amostras recobertas com $\mathrm{SnO}_{2}$, encontrando-se quase sempre agrupadas ao redor de certas cavidades. O conjunto formado por pequenas partículas agrupadas em torno de uma cratera central assemelha-se à mor- 
fologia resultante de um processo de erupção. Estas observações indicam que a solução de ácido fluorídrico penetra pelos poros da camada delgada resultando na dissolução e reprecipitação de pequenas partículas na interface. Como consequência, o depósi-

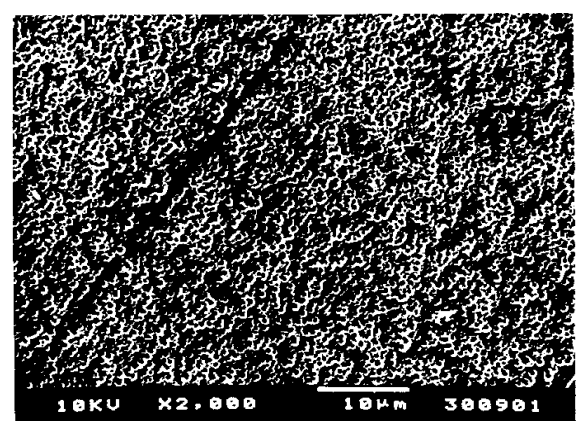

(a)

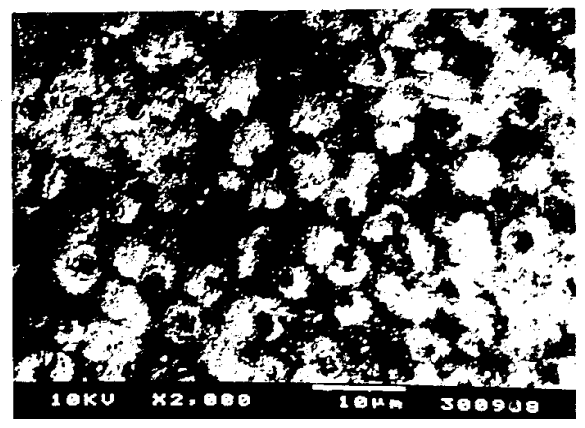

(b)

Figura 6. Micrografias obtidas por microscopia eletrônica de varredura da superficie das amostras submetidas a 25 min de ataque por ácido fluorídrico: a) vidro de borossilicato comercial, b) vidro de borossilicato recoberto com $\mathrm{SnO}_{2}$. to perde a aderência, o que resulta no desprendimento de pequenas porções da camada delgada.

\section{CONCLUSÕES}

- Camadas delgadas transparentes de $\mathrm{SnO}_{2}$ podem ser facilmente depositadas sobre vidros a partir da emersão do substrato em suspensões coloidais aquosas.

- A espessura dos depósitos aumenta de 21 para 90nm quando a velocidade de emersão cresce de 0,1 para $10 \mathrm{~cm} / \mathrm{min}$. $\mathrm{O}$ emprego de velocidades de emersão superiores a $10 \mathrm{~cm} /$ min resulta em depósitos com elevado nível de rugosidade.

- Os vidros recobertos com camadas delgadas de $\mathrm{SnO}_{2}$ apresentam maior resistência ao ataque por ácido fluorídrico.

\section{AGRADECIMENTOS}

Trabalho executado com auxílio financeiro da FAPESP e bolsa de incentivo à pesquisa do CNPq.

\section{REFERÊNCIAS}

1. Davis, M. W.; Smay, G. L.; Wasylyk, J. S.; Am. Ceram. Bull. 1987, 66, 1627.

2. Blunden, S. J.; Cusak, P. A.; Hill, R.; “The Industrial Uses of Tin Chemicals"; Royal Society of Chemistry 1985, London, Chap. 9.

3. Fosse, K. L., Verre 1996, 2, 11.

4. Brinker, C. J.; Scherer, G. W.; "Sol-Gel Science", Academic Press 1990, San Diego, Cap. 8.

5. Hiratsuka, R. S.; Pulcinelli, S. H.; Santilli, C. V.; J. Non. Cryst. Solids 1990, 121, 365.

6. Pulcinelli, S. H.; Santilli, C. V.; Jolivet, J. P.; Tronc, E.; J. Non-Cryst. Solids 1994, 170, 21.

7. Briois, V.; Santilli, C. V.; Pulcinelli, S. H.; Brito, G. E. S.; J. Non-Cryst. Solids 1995, 191, 17.

8. Brower, D. T.; Revay, R. E.; Huang, T. C.; Powder Diffraction 1996, 11, 114.

9. Geoffroy, C.; Campet, G.; Menil, F.; Portier, J.; Salardenne, J.; Coutourier, G.; Active \& Passive Elec. Comp. 1991, 14, 111. 